\title{
La hernia discal lumbar en el niño
}

\author{
Dr. Gonzalo Torrealba M.
}

\section{Lumbar intervertebral dise hemia in a gir}

\begin{abstract}
A case of lumbar (LS-S1) intervertebral disc hernia (LIDH) in an eleven ycar old girl is described. Her main symptoms were lumbar pain, tendernoss in the posterior thigh area and weakness of the flexor muscles of the foot of the right leg. Computed axial tomography (CAT) confirmed the diagnosis and miel og aphy discarded other spine abnormalities. This disease is rare in children and its symptoms and signs are similar to those present in adults. Radiologic examination of the spine including CAT and contrast miclography are very important in order to exclude othet more frequent spinal lesions such as tuberculosis, tumors, piogenic infections and malformations of vertebral structures. Medical management is the treatment of choice, surgery being indicated only in cases of treatment failure or when there is evidence of radicular neurological deficit.
\end{abstract}

(Key words: intervertebral disc hernia, lumbar, first sacral toot compression).

La hernia discal lumbar (HDL), en el niño a diferencia del adulto, es poco frecuente. Entre 0.8 y $3,8 \%$ de las HDL ocurren en personas menores de 21 años. ${ }^{1.2}$ En menores de 15 años es aún más rara: menos del $0.5 \%$ de las HDL operadas. ${ }^{3}$ Estas cifras demuestran que en pediatría rara vez se diagnostica y se trata una HDL. Crecmos entonces de interés -a propósito de un caso clínico- revisar algunos aspectos de esta afección en la infancia.

\section{Caso elínico}

Niồa de 11 ănos de edad. sin antecedentes traumáticos ni mórbidos previos. Consultó por dolor, inicialmente lumbar y luego itradiado por la cara posterior de la extremidad inferior derecha, de un mes de evolución. Tenía intenso dolor lumbociático derecho que limitaba la marcha. Los movimientos de la columna lumbar estaban también limitados por el dolor y había contractu. ra de ta musculatura paravertebral lumbar. No habia signos de déficit sensitivo ni motor. El

1. Neurocirugía. Hospital Gínico Universidad Católica, Santiago. signo de Lasègue era positivo a $10 \mathrm{~s} 60^{\circ}$ en el lado derecho $y$ el reflejo aquiliano ipsilateral estaba abolido. La radiografía de la columna lumbar no mostraba alteraciones. La electromiografja reveló prolongación de la latencia del reflejo $H$. E] hemograma y la velocidad de sedimentación fueron normales.

Un mes más tarde, mediante tomografia computarizada de columna lumbar, sc demostró una hernia discal L5-S1, derecha (figura 1). Con la mielografía lumbar se descartó otras lesiones iłtrarraquídeas y se confirmó la hernia.

Considerando que el tratamiento médico a que fue sometida la paciente durante dos meses había fracasado, pues persistía el dolor y apareció déficit motor en la flexión plantar del pie derecho, se decidió la cirugía. A trayés de una hemisemilaminectomía de L5 derecha se resecó una hernia discal que protruia en el interespacio L5-S1 derecho y comprimía la raíz $\mathrm{S} 1$ de ese lado.

La evolución post-operatoria fue satisfactoria desapareciendo el dolor y la paresia. Controlada algunos meses más tarde estaba asintomática, el examen físico era normal y llevaba una vida normal. 


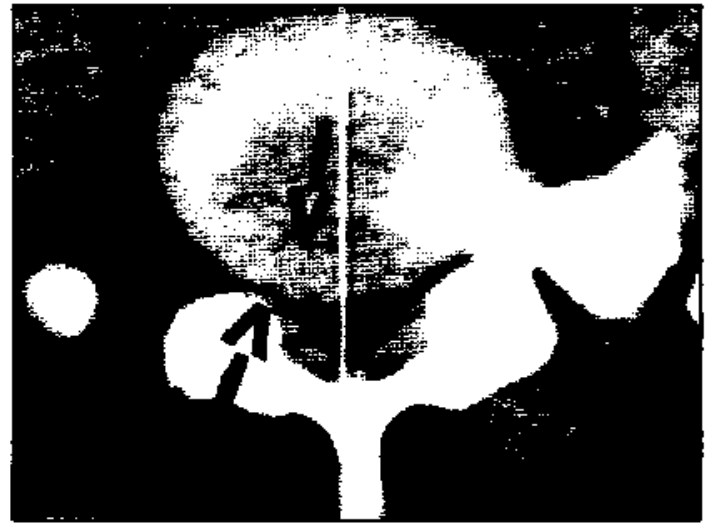

Figura 1: Tomorafía computarizada dcl interespacio LS-SI. Se destaca la protrusión del disco intervertebral en el receso lateral derecho, comprimiendo la raíz $\mathbf{S 1}$ de ese lado.

\section{COMENTARIO}

Es excepcional que una niña de 11 años presente una HDL. Tal es asi que De Orio (1982) analizó cerca de diez mil pacientes operados por esta causa en la Clínica Mayo y encontró que sólo $0,5 \%$ егап menores de 16 años. ${ }^{3}$ Fisher (1981) encontró sólo dos menores de 15 ăios en una serje de 43 pacientes operados que tenían menos de 21 años. ${ }^{4}$ Epstein sólo observó esta afección en jóvenes de 15 a 19 años ${ }^{5}$ y Webb en sólo 3 casos menores de 15 años, de un total de 6.500 pacientes operados de HDL. ${ }^{6}$

La degeneración progresiva del disco parece ser un factor esencial en la génesis de la hernia discal, condición que se produciría con los años. ${ }^{7}$ Esto explicaría lo infrecuente de la HDL en niños. Otro factor importante en la patogenia de csta afección serían los traumatismos repetidos. $5,8,9$ Sin embargo, en sólo un tercio de los niños con HDL existe ese antecedente ${ }^{3}$ y en el caso que comentamos no existía. Se ha sugerido que los traumatismos juegan un rol siempre que existan cambios degenerativos previos, ${ }^{3,5}$ que, aunque raros, podrían verse en niños, a raíz de fallas de la nutrición del disco a causa de insuficiente vascularización. ${ }^{10} \mathrm{El}$ disco, según algunos sería en la infancia irrigado por pequeños vasos provenientes del cartilago de las vértebras adyacentes. Estos vasos desaparecen antes de los 20 anos. ${ }^{7}$ Otros afirman que el disco se nutre por difusión de nutrientes desde el hueso esponjoso de las vértebras adyacentes a través del cartílago. ${ }^{11}$ Con cualquiera de ambos sistemas la nutrición del disco seria bastante precaria $y$ muy vulnerable a cualquier variación de la vascularización, cuya insufíciẹncia produciría rápida degeneración discal. ${ }^{11}$

El cuadro clínico de la HDL en el niño es sjinilar al del adulto, manifestándose por dolor lumbociático; al que pueden agregarse déficits radiculares, motores o sensitivos. Generalmente hay intenso dolor lumbar con limitación de la motilidad de la columna. El signo de Lasègue es positivo y los reflejos aquilianos o rotulianos pueder estar alterados. $3,5,8,9$

La radiografía de columna es indispensable. pues permite descartar lesiones óseas destructivas como son la TBC, tumores vertebrales, espondilitis piógenas que en algún momento de su evolución pueden manifestarse por dolor lumbar y síntomas de lesión radicular. También permite visualizar algunas anomalias vertebrales asociadas como son las espondilolistesis, canal estrecho, vértebras transicionales lumbares, condiciones que parccen ser frecuentes. ${ }^{3,8,9}$

El tratamiento inicial debe ser conservador: reposo, analgésicos y eventual uso de antiinfla. matorios, lográndose un alto porcentaje de éxito en las primeras semanas. Entre 1.368 pacientes que consultaron por dolor lumbar o lumbociá. tica, la cirugía fue necesaria sólo en $50 .^{3}$

La cirugía está indicada, como en nuestro caso, cuando después de un tratamiento médiço bien llevado y por un tiempo adecuado persiste el dolor o bien en presencia de un déficit neuro. lógico radicular. En estas circunstancias la tomografía computarizada de alta resolución tiene su indicación precisa. Su alto rendimiento, ${ }^{12}$ la ausencia de complicaciones y el no ser un examen invasivo en especial en el niño, lo hacen muy recomendable. En nuestro caso se hizo además una mielografía lumbar para descartar lesiones de la "cauda equina", pues la tomografía computarizada no permite diagnosticarlas. Algunos tumores de esa región pueden excepcionalmente manifestarse, al comienzo, de manera similar a la HDL. ${ }^{13}$ Algunos autores sostienen que si la tomografía computarizada muestra claramente una hernia no es necesaria la mielografía. 8

El resultado del tratamiento quirúrgico, ta como en nuestro caso, es generalmente bueno con porcentajes de éxito en el largo plazo que oscilan entre 70 y $90 \% 3,4,8,9$.

\section{RESUMEN}

La hernia de los discos intervertebrales es infrecuente en los niños. Se describe un caso en una paciente de 11 años cuyas principales molestias fueron el dolor lumbar irradiado a la cara posterior del muslo derecho, la limitación dolorosa de la deambulación y los movimientos de la columna, $y$ evidencia de compresión de la primera raíz sacra. La lesión fue demostrada mediante tomografía axial computada y mielografáa, des- 
cartándose otras afecciones más frecuentes de la columna vertebral. El tratamiento de elección es el médico, reservándose la opción quirúrgica sólo para los casos en que éste fracasara en controlar el dolor o existen signos de déficit neurológico radicular.

\section{REFERENCIAS}

1. Rugiveit A.: Juvenile disc her niztion. Acta Orthop Scand $1966 ; 37: 348-356$

2. O'Connell J.E.A.: Intervertebral disc protrusion in childhood and adolescence. J Bone Joint Surg 1960; 47: 611616

3. De Orio J.K., Bianco A.J.: Lumbar disc cxcision in children and adolescents. J Bone Joint Surg 1982; 64 A: $991-996$.

4. Fishcer R.G., Saunders R.L.: Lumbar disc protrusion in children. J Yeurosurg 1981; $54: 480-483$

5. Epstein J.A., Javine L.S.: Herniated lumbar intervertebral disc in tcen-age children. I Neurosurg $1964 ; 21: 1070-1075$.

6. Webb H.J., Slein H.J., Kennedy R.L.J.: Protruded lumbar intervertebral discs in chidren. J A M A 1954: 154: 1153-1154.

7. Drvis CH.: Extradural spinal cord and nerve root compression from benign lesions of the lumbar ares. ln Youmans J.R. ed. Neurological Surgery $2^{\circ}$ ed. Philadelphia W.B. Saunders Co. 1982; 2535-2561.

8. Epstein J.A., Epstein N.E.. Marc J. et al: Lumbar intervertebral disc herniation in teen-age children: rocognition and management of associated anomalies. Spine $1984 ; 9: 427-432$.

9. Kurihara A., Kataoka $O$. Lumbar disc herniation in children and adolescents. Spine 1980: S: 443-445.

10. Plongget $C$., Twerdy $K$. Fischer $J$. et al: Der Klindliche diskusprolaps anhand von vier eigenen Beobachtungen. Neurochirurgia (Stuttgart) 1982; 25: 129-131.

11. Parke $W . W$ : Applied anatomy of the spine. In Rothman R.H., Simeone $\Gamma^{1}$.A. cds. The Spine $2^{\circ}$ ed. Philadelphia: W. B. Saunders Co. 1982; 37-38.

12. Haughton V.M., Eldevik O.P. Magnacs B. et al: A prospective comparison of computed tomography and myelography in the diagnosis of herniated lumbar disc. Radiology 1982; 142: 103-110.

13. Navernouri F.R.C.S.: Neurilemomas of the cauda equina presenting as prolapsed lumbar intervertebral discs. Surg Neurol 1985;23: 187-188. 